\title{
Analysis of a hysteresis-controlled self-oscillating class-D amplifier
}

\author{
Stephen M. Cox*, Jun YU ${ }^{\dagger}$, WANG Ling GoHªnd Meng Tong TAN ${ }^{\ddagger}$ \\ ${ }^{*}$ School of Mathematical Sciences, University of Nottingham, \\ University Park, Nottingham NG7 2RD, UK \\ ${ }^{\dagger}$ Institute of Microelectronics, Agency for Science, Technology and Research, \\ 11 Science Park Road, Singapore 117685, Singapore
}

$\ddagger$ School of Electrical and Electronic Engineering, Nanyang Technological University,
Singapore 639798, Singapore

This paper gives the first systematic perturbation analysis of the audio distortion and mean switching period for a self-oscillating class-D amplifier. Explicit expressions are given for all the principal components of audio distortion, for a general audio input signal; the specific example of a sinusoidal input is also discussed in some detail, yielding an explicit closed-form expression for the total harmonic distortion (THD). A class-D amplifier works by converting a low-frequency audio input signal to a high-frequency train of rectangular pulses, whose widths are slowly modulated according to the audio signal. The audiofrequency components of the pulse-train are designed to agree with those of the audio signal. In many varieties of class-D amplifier, the pulse-train is generated using a carrier wave of fixed frequency, well above the audio range. In other varieties, as here, there is no such fixed-frequency clock, and the local frequency of the pulse-train varies in response to the audio input. Such self-oscillating designs pose a particular challenge for comprehensive mathematical modelling; we show that in order to properly account for the local frequency variations, a warped-time transformation is necessary. The systematic nature of our calculation means it can potentially be applied to a range of other self-oscillating topologies. Our results for a general input allow ready calculation of distortion diagnostics such as the intermodulation distortion (IMD), which prior analyses, based on sinusoidal input, cannot provide.

Keywords: class-D amplifier, mathematical model, astable integrating modulator, hysteresis-controlled self-oscillating modulator, hysteresis comparator.

\section{Introduction}

Class-D amplifiers are nowadays used in a wide variety of consumer products (Berkhout \& Dooper, 2010); in particular, their increasing adoption in mobile electronic devices derives from their high efficiency, which reduces heat generation and extends battery life. At the heart of a class-D amplifier is the conversion of an audio signal (of relatively low frequency) to a train of rectangular pulses of much higher frequency. The widths of these pulses are modulated according to the audio signal in such a way that the low-frequency components in the pulse-train correspond closely to the audio signal itself. This technique is pulse-width modulation (PWM) (Black, 1953).

Conventional PWM is achieved by comparing the audio signal (or some other signal in the circuit) with a high-frequency carrier wave (typically a sawtooth or triangular wave); the output pulse-train then switches whenever the difference between the two signals changes sign. In such designs, the quality of the audio reproduction depends crucially on the precision with which the slopes of the carrier wave can be made linear (Tan et al., 2003); this requirement for a highly linear, high-frequency carrier wave poses a particular technological challenge. Furthermore, the presence of a fixed-frequency carrier wave gives rise to strong high-frequency peaks in the power spectrum of the output, which can lead to undesirable 
electromagnetic interference (EMI) affecting nearby equipment.

In contrast, the self-oscillating variety of class-D amplifier, as treated in this paper, obviates the need for a carrier wave (Green \& Williams, 1992; Høyerby \& Andersen, 2009; Lu \& Gharpurey, 2011; Piessens \& Steyaert, 2005; Poulsen \& Andersen, 2004; Putzeys, 2005, 2009; Yu et al., 2009). It is thus potentially advantageous for low-cost implementation, since it eliminates the requirement for a separate high-precision carrier-wave generator. A further benefit is that the frequency of the output pulse-train tends to vary with the audio signal, so the high-frequency spectrum of the output is spread, with consequent reduction in EMI. Power losses through switching can be reduced, since the switching frequency drops at peaks in the magnitude of the input signal. Lu \& Gharpurey (2011) also observe that their self-oscillating class-D amplifier suffers less significant harmonic distortion than a conventional carrier-wave-based design. It may be worthwhile to note the variety of terminology used in the engineering literature to describe related self-oscillating designs; such terms include the astable integrating multivibrator (AIM) (Poulsen \& Andersen, 2004) and the sigma-delta modulator (Green \& Williams, 1992).

Although self-oscillating class-D amplifiers are widely used, the corresponding mathematical analysis is relatively limited. Two principal reasons for the difficulty in providing a comprehensive mathematical theory are (i) the presence of negative feedback (introduced to suppress, for example, the influence of power-supply imperfections) and (ii) the presence of slow modulations in the pulse-train frequency. Prior analyses (e.g., Green \& Williams, 1992; Høyerby \& Andersen, 2009; Lu \& Gharpurey, 2011) generally derive results for a constant (i.e., DC) input, $s_{0}$, and infer analogous results for a time-varying audio input, $s(t)$, by substituting $s(t)$ for $s_{0}$ in the constant-input results, then making further approximations. The use of such a quasi-static assumption is understandable, because the audio signal typically varies only slightly over one period of the switching of the pulse-train, and it seems to provide a reasonable "leading-order" approximation to the operation of the device, albeit after some parameter fitting (Lu \& Gharpurey, 2011). Other analyses (Chang \& Tan, 2011; Piessens \& Steyaert, 2005) approximate the behaviour of the comparator that generates the rectangular-wave output. However, it is difficult to quantify the effects of the various (often rather ad hoc) approximations in prior treatments, and it is unclear that all contributions to practically important quantities such as the total harmonic distortion (THD) have been properly accounted for. Finally, because such approaches are not entirely systematic, it is unclear how they can be extended to different amplifier topologies.

In this work, we present a systematic analysis for a simple, first-order, self-oscillating class-D amplifier with a single negative-feedback path and with a genuinely time-varying input. The architecture of this self-oscillating amplifier is similar to that of a first-order continuous-time sigma-delta modulator (Green \& Williams, 1992). Our approach is based on perturbation methods, the small parameter $\varepsilon$ being proportional to the ratio of typical audio frequencies to typical switching frequencies; it requires significant modification to the approach used in our previous treatments of class-D amplifiers (Cox \& Candy, 2005; Cox \& Mouton, 2015; Cox et al., 2011, 2013; Yu et al., 2012), all of which have concerned fixed-clock-frequency designs. Specifically, it is necessary to introduce a "warped-time" transformation to account for slow modulation of the switching frequency. Furthermore, the form of this transformation must be chosen so that the final, demodulation stage of the calculation can be carried out straightforwardly.

Our results comprise a set of closed-form expressions for (i) the mean period of the output switching and (ii) the audio components of the amplifier output, from which the distortion characteristics may be determined. In particular, by construction the audio output expression guarantees to give all components of distortion at each order in $\varepsilon$ (by contrast, the ad hoc nature of previous approaches does not allow a corresponding statement to be made there). A significant achievement of the present work is that such 
expressions are given for a general audio input. While results are illustrated for the important test case of a sinusoidal input, from which the THD is calculated, the general expression provides a valuable tool - not previously available - for engineers who wish to calculate measures of distortion beyond just the THD, such as the intermodulation distortion (IMD) (Cox et al., 2011, 2013; Guo et al., 2014; Metzler, 1993; Yu et al., 2012) that occurs when the input contains two or more frequencies.

The importance of quantifying the audio distortion is clear. But, from a practical perspective, it is also important to have an accurate relation between switching frequency and input signal, particularly in stereo applications, where a difference in switching frequency between the channels can inject lowfrequency intermodulation products into the audio band (Pillonnet et al., 2008). In addition, a decrease in carrier frequency can significantly worsen the performance of the amplifier as the output power level increases (Poulsen \& Andersen, 2005), or even cause stability problems in a high-order design. Our analysis allows one to determine (at least in principle) the signal-frequency relation to any order in $\varepsilon$, where previous approaches are limited to a leading-order estimate.

This paper is organised as follows. In Section 2, we formulate our mathematical model for a firstorder, self-oscillating class-D amplifier. This model is solved by perturbation methods in Section 3, incorporating the warped-time transformation; the main results for the audio output and the mean switching period are presented in Section 3.3. Our asymptotic results are compared with numerical simulations in Section 4, with excellent agreement. We compare our results with those of Lu \& Gharpurey (2011), which are based on a quasi-static approximation, in Section 5. Finally, we give our conclusions in Section 6.

\section{Mathematical formulation}

In this section, we set up our mathematical model for the self-oscillating amplifier. We emphasise the crucial distinction between this work and the usual engineering approach, which is that at no point do we adopt a quasi-static approximation; instead we take full account of the time-variation of the audio input signal.

Figure 1 shows the specific circuit design examined in this paper. The output is a high-frequency rectangular pulse-train $g(t)$, scaled so that $g(t)$ takes the values \pm 1 . The widths of the output pulses are modulated in such a way that the low-frequency components of $g(t)$ should closely reproduce those of $-s(t)$, where $s(t)$ is the audio input signal, scaled so that $-1<s(t)<1$. (The minus sign difference between input and output generates no audible consequences.) The design in Fig. 1 incorporates negative feedback: the output and input are summed, and this "error" signal is integrated to give $h(t)$, so that

$$
\frac{\mathrm{d} h}{\mathrm{~d} t}=-c(s(t)+g(t)),
$$

where $c$ is a positive constant. In view of the need to integrate $s(t)$ when solving (1), we introduce $f(t)$ such that

$$
s(t)=f^{\prime}(t) .
$$

The integrated error signal is then summed with a multiple of the output and fed to the noninverting input of a comparator, whose inverting input is fixed at 0 . The output of the comparator takes the values \pm 1 according to which of its inputs has the larger signal: specifically,

$$
g(t)= \begin{cases}+1 & \text { if } h(t)+H g(t)>0, \\ -1 & \text { if } h(t)+H g(t)<0,\end{cases}
$$




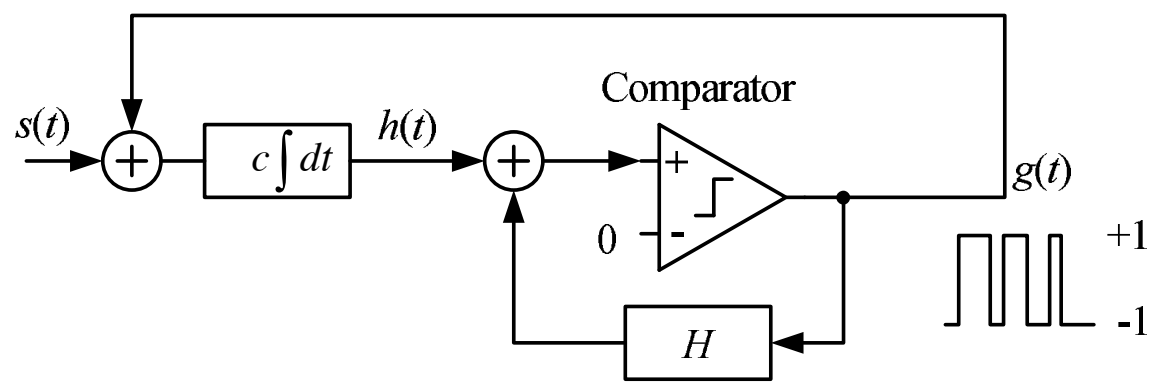

FIG. 1. Hysteresis-controlled, self-oscillating, first-order, class-D amplifier. See text for details.

where $H$ is a positive constant. The positive feedback path surrounding the comparator generates hysteresis, and $H$ is termed the hysteresis factor (Lu \& Gharpurey, 2011). It is readily determined that in the absence of any audio input (i.e., for $s(t) \equiv 0$ ) the device oscillates with quiescent switching period

$$
T_{0} \equiv \frac{4 H}{c} .
$$

More generally, if the input is constant, say $s(t) \equiv s_{0}$, then the switching period is readily found to be $T_{0} /\left(1-s_{0}^{2}\right)$ (cf. Green \& Williams, 1992; Poulsen \& Andersen, 2005).

We denote the times at which the output switches by $A_{n}$ and $B_{n}$, for integers $n$, with

$$
g(t)= \begin{cases}+1 & \text { for } B_{n}<t<A_{n+1}, \\ -1 & \text { for } A_{n}<t<B_{n} .\end{cases}
$$

Then by integrating (1) separately over the time intervals $A_{n}<t<B_{n}$ and $B_{n}<t<A_{n+1}$ and imposing the switching conditions

$$
h\left(A_{n}\right)+H=0 \quad \text { and } \quad h\left(B_{n}\right)-H=0,
$$

we find that the amplifier is governed by the nonlinear difference equations

$$
\begin{aligned}
-f\left(B_{n}\right)+f\left(A_{n}\right)+B_{n}-A_{n}=2 H / c, \\
f\left(A_{n+1}\right)-f\left(B_{n}\right)+A_{n+1}-B_{n}=2 H / c .
\end{aligned}
$$

Note that these expressions hold for a general (that is, not necessarily sinusoidal) audio input signal $s(t)$. Solution of these equations and determination of the corresponding audio components of the output form the subject of the remainder of this paper.

\section{Solving the model}

\subsection{Preliminaries}

It is helpful in solving (5) and (6) to write $A_{n}$ and $B_{n}$ in the form

$$
A_{n}=\left(n+\alpha_{n}\right) T, \quad B_{n}=\left(n+\beta_{n}\right) T,
$$


where $T$ is some nominal switching period, which will be discussed further below. For amplifiers with a fixed-frequency carrier-wave, $T$ is simply the period of that wave and, furthermore, $\alpha_{n}$ and $\beta_{n}$ each lie in the interval $(0,1)$, for all $n$. In the present, self-oscillating case, however, the "local switching period" (which may be defined, for example, as $A_{n+1}-A_{n}$ ) varies according to the input signal. Thus (since $T$ is constant) $\alpha_{n}$ and $\beta_{n}$ are no longer constrained to lie in $(0,1)$; indeed, since the audio signal varies slowly compared with the switching, $\alpha_{n}$ and $\beta_{n}$ may be expected to become large, as the local switching period drifts (for many switching periods) from its mean.

Before attempting to solve (5) and (6) for $A_{n}$ and $B_{n}$, it is useful to consider the final, demodulation stage of the calculation, which involves extracting the audio components of the amplifier output from these switching times. This will motivate the efficient (but otherwise rather unintuitive) form in which we seek expressions for the $A_{n}$ and $B_{n}$.

We begin by noting that the amplifier output takes the form (Cox, 2009; Cox \& Candy, 2005; Cox et al., 2011)

$$
g(t)=\sum_{n=-\infty}^{\infty}\left\{\psi\left(t ; B_{n}, A_{n+1}\right)-\psi\left(t ; A_{n}, B_{n}\right)\right\}
$$

where the "top-hat" function $\psi$ is given by

$$
\psi\left(t ; t_{1}, t_{2}\right)= \begin{cases}1 & \text { for } t_{1}<t<t_{2} \\ 0 & \text { otherwise }\end{cases}
$$

Applying the Poisson resummation identity,

$$
\sum_{n=-\infty}^{\infty} \Phi(n)=\sum_{n=-\infty}^{\infty} \int_{-\infty}^{\infty} \mathrm{e}^{2 \pi \mathrm{i} n \tau} \Phi(\tau) \mathrm{d} \tau
$$

to $(8)$ yields

$$
g(t)=\sum_{n=-\infty}^{\infty} \int_{-\infty}^{\infty} \mathrm{e}^{2 \pi \mathrm{i} n \tau}\{\psi(t ; B(\tau), A(\tau+1))-\psi(t ; A(\tau), B(\tau))\} \mathrm{d} \tau
$$

where the smooth functions $A$ and $B$ are such that

$$
A(n)=A_{n}, \quad B(n)=B_{n} .
$$

Note that, while only these sampled values of $A$ and $B$ have physical significance, the mathematical analysis below is rendered considerably more straightforward by seeking expressions for $A$ and $B$ for all real values of their arguments.

To make progress in evaluating (11), we note (7) and suppose that we have written the variables $\alpha_{n}$ and $\beta_{n}$ in the form

$$
\alpha_{n}=a\left(A_{n}\right), \quad \beta_{n}=b\left(B_{n}\right),
$$

for some functions $a$ and $b$. This rather strange-looking step is motivated by our previous analysis of natural sampling in an open-loop class-D amplifier (Cox, 2009; Cox \& Candy, 2005); its utility will become clear below, when we derive a formula for the audio components of the output. We extend the definitions in (7) and (13) in the natural way, so that

$$
A(\tau)=(\tau+a(A(\tau))) T \quad \text { and } \quad B(\tau)=(\tau+b(B(\tau))) T
$$

for all real $\tau$. 
In evaluating the right-hand side of (11), we note that $\psi(t ; A(\tau), B(\tau))=0$ outside the interval $A(\tau)<t<B(\tau)$. In view of the preceding discussion, the lower limit, $t=A(\tau)$, may equivalently be written $t=(\tau+a(t)) T$, so that $\tau=t / T-a(t)$. Likewise, the upper limit, $t=B(\tau)$, may be written $\tau=t / T-b(t)$. Similar considerations apply to the term in (11) involving $\psi(t ; B(\tau), A(\tau+1))$, with the limit $t=A(\tau+1)$ being equivalent to $\tau=t / T-1-a(t)$. The amplifier output thus takes the form

$$
g(t)=\sum_{n=-\infty}^{\infty} \int_{-\infty}^{\infty} \mathrm{e}^{2 \pi \mathrm{i} n \tau}\{\psi(\tau ; t / T-1-a(t), t / T-b(t))-\psi(\tau ; t / T-b(t), t / T-a(t))\} \mathrm{d} \tau .
$$

Hence

$$
g(t)=1-2(b(t)-a(t))+\sum_{n=-\infty}^{\infty} \frac{\mathrm{e}^{2 \pi \mathrm{i} n t / T}}{n \pi \mathrm{i}}\left(\mathrm{e}^{-2 \pi \mathrm{i} n b(t)}-\mathrm{e}^{2 \pi \mathrm{i} n a(t)}\right),
$$

where the prime indicates that the term $n=0$ is omitted from the sum. If we suppose (as is the case in practice) that the frequencies associated with the switching of the output are significantly above the audio band, then the audio component of the output, $g_{a}(t)$, corresponds to omitting the sum from (14), so that

$$
g_{a}(t)=1-2(b(t)-a(t)) .
$$

This expression for the audio output is particularly simple, once the functions $a$ and $b$ introduced in (13) have been determined.

\subsection{Solving the model for the output switching times}

We thus seek to solve the governing difference equations (5) and (6) to give the switching times according to implicit equations of the form

$$
A_{n}=\left(n+a\left(A_{n}\right)\right) T, \quad B_{n}=\left(n+b\left(B_{n}\right)\right) T
$$

since then the audio output follows immediately from (15). In doing so, we introduce a "typical" (angular) frequency $\omega$, for the audio signal. Thus we adopt a scaled time $\theta=\omega t$ and write the input signal as

$$
s(t)=S(\theta) .
$$

Correspondingly, we write $f(t)=F(\theta) / \omega$ so that $S(\theta)=F^{\prime}(\theta)$.

Then the switching equations (5) and (6) become the nonlinear difference equations

$$
\begin{array}{r}
-F\left(\omega B_{n}\right)+F\left(\omega A_{n}\right)+\omega B_{n}-\omega A_{n}=\varepsilon / 2, \\
F\left(\omega A_{n+1}\right)-F\left(\omega B_{n}\right)+\omega A_{n+1}-\omega B_{n}=\varepsilon / 2,
\end{array}
$$

where we have introduced

$$
\varepsilon=\frac{4 H \omega}{c} \ll 1 .
$$

We see that $\varepsilon=\omega T_{0}$, where $T_{0}$ is the quiescent switching period, given in (3); the smallness of the parameter $\varepsilon$, which is crucial to the remainder of our analysis, embodies the assumed disparity between audio and switching frequencies.

To find $A_{n}$ in the requisite form, we introduce into (17) and (18) the quantities

$$
\eta_{n}=\omega A_{n}, \quad \gamma_{n}=\omega\left(B_{n}-A_{n}\right), \quad \delta_{n}=\omega\left(A_{n+1}-A_{n}\right),
$$


so that (17) and (18) become

$$
\begin{array}{r}
-F\left(\eta_{n}+\gamma_{n}\right)+F\left(\eta_{n}\right)+\gamma_{n}=\varepsilon / 2, \\
F\left(\eta_{n}+\delta_{n}\right)-F\left(\eta_{n}+\gamma_{n}\right)+\delta_{n}-\gamma_{n}=\varepsilon / 2 .
\end{array}
$$

By standard perturbation techniques, (21) may be solved for $\gamma_{n}$ in powers of the small parameter $\varepsilon$ to give

$$
\gamma_{n}=\frac{\varepsilon}{2\left(1-S\left(\eta_{n}\right)\right)}+\frac{\varepsilon^{2} S^{\prime}\left(\eta_{n}\right)}{8\left(1-S\left(\eta_{n}\right)\right)^{3}}+\frac{\varepsilon^{3}\left(3\left(S^{\prime}\left(\eta_{n}\right)\right)^{2}+S^{\prime \prime}\left(\eta_{n}\right)\left(1-S\left(\eta_{n}\right)\right)\right.}{48\left(1-S\left(\eta_{n}\right)\right)^{5}}+O\left(\varepsilon^{4}\right)
$$

Given this solution for $\gamma_{n}$, we may then solve (22) similarly to give

$$
\delta_{n}=\frac{\varepsilon}{1-S^{2}\left(\eta_{n}\right)}+\frac{\varepsilon^{2} S^{\prime}\left(\eta_{n}\right)\left(S^{2}\left(\eta_{n}\right)+4 S\left(\eta_{n}\right)-1\right)}{4\left(1-S^{2}\left(\eta_{n}\right)\right)^{3}}+O\left(\varepsilon^{3}\right)
$$

The next step is to find $\eta_{n}$, which then immediately provides $A_{n}$ from (20). To do so, we note, from (20), that $\eta_{n}$ satisfies the difference equation

$$
\eta_{n+1}-\eta_{n}=\delta_{n}
$$

To solve this equation, we introduce smooth functions $\eta$ and $\delta$ such that

$$
\eta(\varepsilon n)=\eta_{n}, \quad \delta(\varepsilon n)=\delta_{n} ;
$$

then the difference equation (24) becomes

$$
\eta(\tau+\varepsilon)-\eta(\tau)=\delta(\tau)
$$

with, from (23),

$$
\delta(\tau) \sim \frac{\varepsilon}{1-S^{2}(\eta(\tau))}+\frac{\varepsilon^{2} S^{\prime}(\eta(\tau))\left(S^{2}(\eta(\tau))+4 S(\eta(\tau))-1\right)}{4\left(1-S^{2}(\eta(\tau))\right)^{3}} .
$$

The most natural approach would now arguably be to solve (26) for $\eta(\tau)$ as a power series in $\varepsilon$. However, it turns out that this does not yield $A_{n}$ in the required form. So instead we introduce the inverse function $y$ (so that $y(\eta(\tau))=\tau$ ), in terms of which (26) becomes

$$
\tau+\varepsilon=y(\eta(\tau)+\delta(\tau))
$$

which may be solved to give $y$ in the form

$$
y(\eta)=P \eta+y_{0}(\eta)+\varepsilon y_{1}(\eta)+\varepsilon^{2} y_{2}(\eta)+\varepsilon^{3} y_{3}(\eta)+O\left(\varepsilon^{4}\right),
$$

where the $y_{n}(\eta)$ are all bounded functions of $\eta$.

It is worth pausing to interpret (28). If we set $\tau=\varepsilon n$ and, correspondingly, $\eta=\omega A_{n}$, we find, from (20) and (25) that (28) becomes

$$
\varepsilon n=y\left(\omega A_{n}\right)=P \omega A_{n}+y_{0}\left(\omega A_{n}\right)+\varepsilon y_{1}\left(\omega A_{n}\right)+\varepsilon^{2} y_{2}\left(\omega A_{n}\right)+\cdots
$$


and hence

$$
A_{n}=\frac{\varepsilon}{P \omega}\left(n-\varepsilon^{-1} y_{0}\left(\omega A_{n}\right)-y_{1}\left(\omega A_{n}\right)-\varepsilon y_{2}\left(\omega A_{n}\right)-\cdots\right) .
$$

This is precisely the implicit definition of $A_{n}$ as required in (16).

It is remarkable that the $y_{n}$ may next be determined, in closed form, for a general audio input. We find, after much algebra, that

$$
\begin{aligned}
& y_{0}(\eta)=\int\left\langle S^{2}(\eta)\right\rangle-S^{2}(\eta) \mathrm{d} \eta \\
& y_{1}(\eta)=\frac{S(\eta)}{4} \\
& y_{2}(\eta)=\frac{S(\eta) S^{\prime}(\eta)}{24\left(1-S^{2}(\eta)\right)}+\frac{1}{48} \int\left\langle\frac{\left(S^{\prime}(\eta)\right)^{2}}{1-S^{2}(\eta)}\right\rangle-\frac{\left(S^{\prime}(\eta)\right)^{2}}{1-S^{2}(\eta)} \mathrm{d} \eta \\
& y_{3}(\eta)=-\frac{S^{\prime \prime}(\eta)}{192\left(1-S^{2}(\eta)\right)}-\frac{S(\eta)\left(S^{\prime}(\eta)\right)^{2}}{96\left(1-S^{2}(\eta)\right)^{2}} .
\end{aligned}
$$

Here $\langle\cdots\rangle$ denotes the average value. Note that $y_{n}(\eta)$ is odd or even in $S$ according to whether the index $n$ is, respectively, odd or even. The constant $P$ turns out to be given by

$$
P=1-\left\langle S^{2}(\eta)\right\rangle-\frac{\varepsilon^{2}}{48}\left\langle\frac{\left(S^{\prime}(\eta)\right)^{2}}{1-S^{2}(\eta)}\right\rangle+O\left(\varepsilon^{4}\right) .
$$

In the special case of sinusoidal input, with

$$
s(t)=s_{0} \sin \omega t,
$$

hence $S(\eta)=s_{0} \sin \eta$, these expressions become

$$
\begin{aligned}
& y_{0}(\eta)=\frac{1}{4} s_{0}^{2} \sin 2 \eta \\
& y_{1}(\eta)=\frac{s_{0} \sin \eta}{4}, \\
& y_{2}(\eta)=\frac{\left(1-s_{0}^{2}\right)^{1 / 2}\left\{\tan ^{-1}\left(\left(1-s_{0}^{2}\right)^{1 / 2} \tan \eta\right)-\eta\right\}}{48}+\frac{s_{0}^{2} \sin 2 \eta}{48\left(1-s_{0}^{2} \sin ^{2} \eta\right)} \\
& y_{3}(\eta)=\frac{\left(1-2 s_{0}^{2}+s_{0}^{2} \sin ^{2} \eta\right) s_{0} \sin \eta}{192\left(1-s_{0}^{2} \sin ^{2} \eta\right)^{2}}
\end{aligned}
$$

We have thus succeeded in obtaining $A_{n}$ in the form (16), with mean switching period

$$
T=\frac{\varepsilon}{P \omega} \sim \frac{4 H}{c\left\langle 1-s^{2}(t)-(1 / 3)(H / c)^{2}\left(s^{\prime}(t)\right)^{2} /\left(1-s^{2}(t)\right)\right\rangle}
$$

and

$$
a\left(A_{n}\right)=-\varepsilon^{-1} y_{0}\left(\omega A_{n}\right)-y_{1}\left(\omega A_{n}\right)-\varepsilon y_{2}\left(\omega A_{n}\right)-\cdots .
$$

Having determined $a\left(A_{n}\right)$, we now turn to finding $b\left(B_{n}\right)$. This may largely be accomplished by recycling the calculation above. We begin by substituting

$$
\phi_{n}=\omega B_{n}, \quad \xi_{n}=\omega\left(A_{n+1}-B_{n}\right), \quad \zeta_{n}=\omega\left(B_{n+1}-B_{n}\right)
$$


in (17) and (18) and manipulating the results to give

$$
\begin{array}{r}
F\left(\phi_{n}+\xi_{n}\right)-F\left(\phi_{n}\right)+\xi_{n}=\varepsilon / 2, \\
-F\left(\phi_{n}+\zeta_{n}\right)+F\left(\phi_{n}+\xi_{n}\right)+\zeta_{n}-\xi_{n}=\varepsilon / 2 .
\end{array}
$$

By comparing (41) with (21), we see that if $\gamma_{n}=\Gamma\left(S\left(\eta_{n}\right)\right)$ then $\xi_{n}=\Gamma\left(-S\left(\phi_{n}\right)\right)$. Furthermore, by comparing (42) with (22), we see that if $\delta_{n}=\Delta\left(S\left(\eta_{n}\right)\right)$ then $\zeta_{n}=\Delta\left(-S\left(\phi_{n}\right)\right)$. Thus $S$ is replaced by $-S$ when moving from the calculation of $a$ to the calculation of $b$. Furthermore, at each stage in the calculation of $b(t)$ as a series in powers of $\varepsilon$, we integrate with respect to $t$, and hence introduce a constant of integration; thus we see that

$$
b(t ; S)=a(t ;-S)+\text { constant }
$$

By substituting $S=0$ in this expression, in which case $a$ and $b$ are constant functions, with $b(t)=$ $a(t)+1 / 2$, we obtain the value of the constant of integration as $1 / 2$. Thus

$$
b\left(B_{n}\right)=-\varepsilon^{-1} y_{0}\left(\omega B_{n}\right)+\frac{1}{2}+y_{1}\left(\omega B_{n}\right)-\varepsilon y_{2}\left(\omega B_{n}\right)+\varepsilon^{2} y_{3}\left(\omega B_{n}\right)-\cdots .
$$

\subsection{Results for audio output and mean switching period}

Having calculated the switching times of the output, we now construct the audio component of the output, which is, from (15),

$$
g_{a}(t)=1-2(b(t)-a(t))=-4 y_{1}(\omega t)-4 \varepsilon^{2} y_{3}(\omega t)+O\left(\varepsilon^{4}\right) .
$$

For a sinusoidal input of the form (34), the audio output thus becomes

$$
\begin{aligned}
g_{a}(t) & \sim-s_{0} \sin \omega t-\left(\frac{H \omega}{c}\right)^{2} \frac{s_{0} \sin \omega t\left(1-2 s_{0}^{2}+s_{0}^{2} \sin ^{2} \omega t\right)}{3\left(1-s_{0}^{2} \sin ^{2} \omega t\right)^{2}} \\
& =-s_{0} \sin \omega t-\left(\frac{H \omega}{c}\right)^{2} \sum_{m=0}^{\infty} C_{2 m+1} \sin ((2 m+1) \omega t),
\end{aligned}
$$

where

$$
C_{2 m+1}=\frac{2(2 m+1)\left(\left(1-s_{0}^{2}\right)^{1 / 2}-1\right)^{m} s_{0}}{3\left(\left(1-s_{0}^{2}\right)^{1 / 2}+1\right)^{m+1}},
$$

and a derivation of these Fourier-series coefficients is given in the appendix. We note that the leading distortion terms are thus quadratic in the audio frequency $\omega$.

For a general input $s(t)$, the audio output is, correspondingly,

$$
\begin{aligned}
g_{a}(t) & \sim-s(t)+\frac{H^{2}}{3 c^{2}}\left(\frac{s^{\prime \prime}(t)}{1-s^{2}(t)}+\frac{2 s(t)\left(s^{\prime}(t)\right)^{2}}{\left(1-s^{2}(t)\right)^{2}}\right) \\
& =-s(t)+\frac{H^{2}}{3 c^{2}} \frac{\mathrm{d}^{2}}{\mathrm{~d} t^{2}} \tanh ^{-1} s(t) .
\end{aligned}
$$

The expressions in (45)/(46) and (48)/(49) represent the culmination of the calculation described above; they provide in closed-form the leading components of distortion for the self-oscillating class-D amplifier in Fig. 1. 
Finally, the leading-order mean switching period satisfies

$$
T \sim \frac{4 H}{\left\langle 1-s^{2}(t)\right\rangle c}
$$

which, in the case of sinusoidal input $s(t)=s_{0} \sin \omega t$, becomes the known result (Green \& Williams, 1992; Lu \& Gharpurey, 2011)

$$
T \sim \frac{4 H}{\left(1-\frac{1}{2} s_{0}^{2}\right) c} .
$$

Our analysis then provides the first correction to this result, which gives, from (39),

$$
T \sim \frac{4 H}{\left(1-\frac{1}{2} s_{0}^{2}\right) c}\left\{1+\frac{\omega^{2} H^{2} / c^{2}}{3\left(1-\frac{1}{2} s_{0}^{2}\right)}\left(1-\left(1-s_{0}^{2}\right)^{1 / 2}\right)\right\} .
$$

\subsection{Total harmonic distortion}

For the case of sinusoidal input, a common diagnostic of the audio fidelity is the total harmonic distortion, given here to $O\left(\varepsilon^{2}\right)$ by

$$
\mathrm{THD}_{\%}=\frac{(H \omega / c)^{2} \sqrt{C_{3}^{2}+C_{5}^{2}+\cdots}}{s_{0}} \times 100 \% .
$$

where the coefficients $C_{3}, \ldots$ are given in (47). It turns out that the infinite sum in this expression may be evaluated in closed form, giving

$$
\mathrm{THD} \%=\frac{H^{2} \omega^{2}}{c^{2}} \sqrt{\frac{1+\left(1-s_{0}^{2}\right)^{2}}{18\left(1-s_{0}^{2}\right)^{3 / 2}}-\frac{4}{9\left(1+\left(1-s_{0}^{2}\right)^{1 / 2}\right)^{2}}} \times 100 \% .
$$

In practice, however, one would truncate the sum to include only those harmonics within the range of human hearing (typically $20 \mathrm{~Hz}-20 \mathrm{kHz}$ ); thus the number of terms included would depend on the audio frequency.

\section{Comparison with simulations}

In this section, we compare our analytical results with simulations using MATLAB Simulink. The parameter values chosen are $c=10^{5}$ and $H=0.05$. In addition, we present some analytical results for a conventional (i.e., carrier-wave-based) first-order PWM design for comparison purposes. The switching frequency of the conventional design is set to $500 \mathrm{kHz}$, which is the idle switching frequency of the self-oscillating design (assuming, as throughout this section, that we adopt seconds as our unit of time).

Figure 2 shows the average switching frequency $F_{s} \equiv T^{-1}$ for a sinusoidal input as in (34), against the modulation index, $s_{0}$. We take $s_{0} \in[0.1,0.9]$ and set the input signal frequency to $5 \mathrm{kHz}$ (so $\omega=10^{4} \pi$ ). As illustrated in Fig. 2, the agreement between the analytical results and the MATLAB simulations is excellent.

Figure 3 shows the THD performance against the modulation index. Again, we take $s_{0} \in[0.1,0.9]$ and set the input frequency to $5 \mathrm{kHz}$. The THD results are calculated based on all the harmonics within 


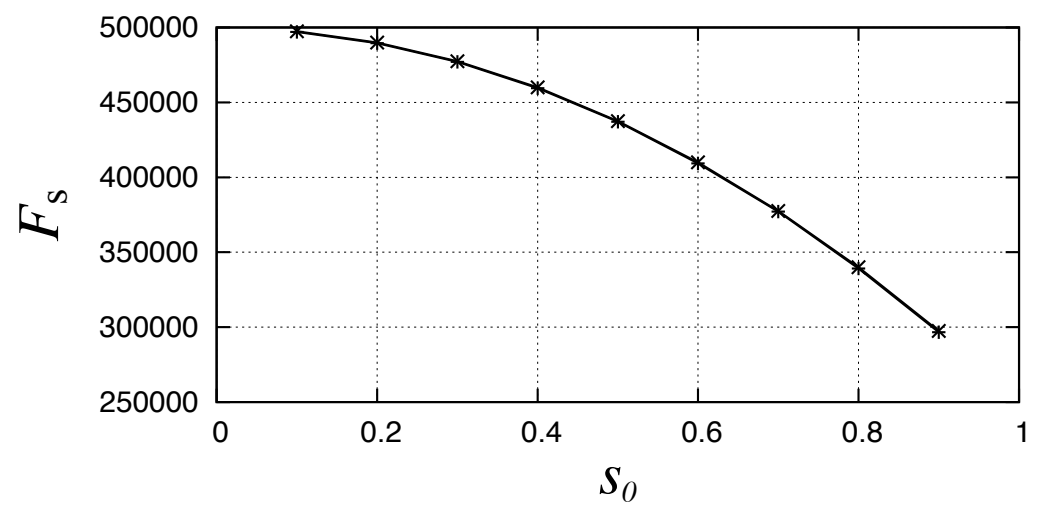

FIG. 2. Average switching frequency $F_{\mathrm{s}}$ against modulation index $s_{0}$ for a sinusoidal input signal of frequency $5 \mathrm{kHz}$. Plotted are results from MATLAB simulations $(+$ symbol) and analysis $(\times$ symbol), using $(51)$. The two sets of results are indistinguishable on the plot.

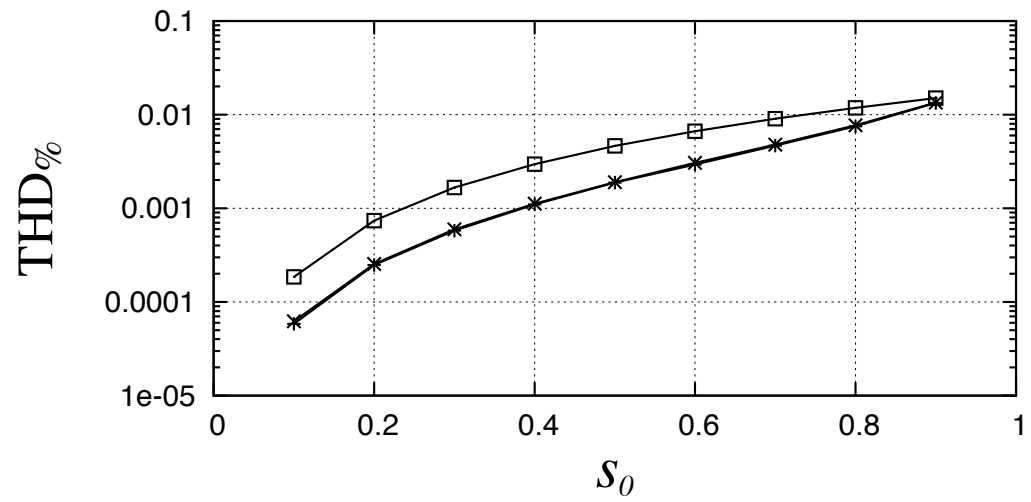

FIG. 3. THD $\%$ against modulation index $s_{0}$ for a sinusoidal input signal of frequency $5 \mathrm{kHz}$. Results from MATLAB simulation and analysis are, respectively, indicated by the symbols + and $\times$ (these results are indistinguishable on the plot); the symbol $\square$ indicates results for conventional PWM. 


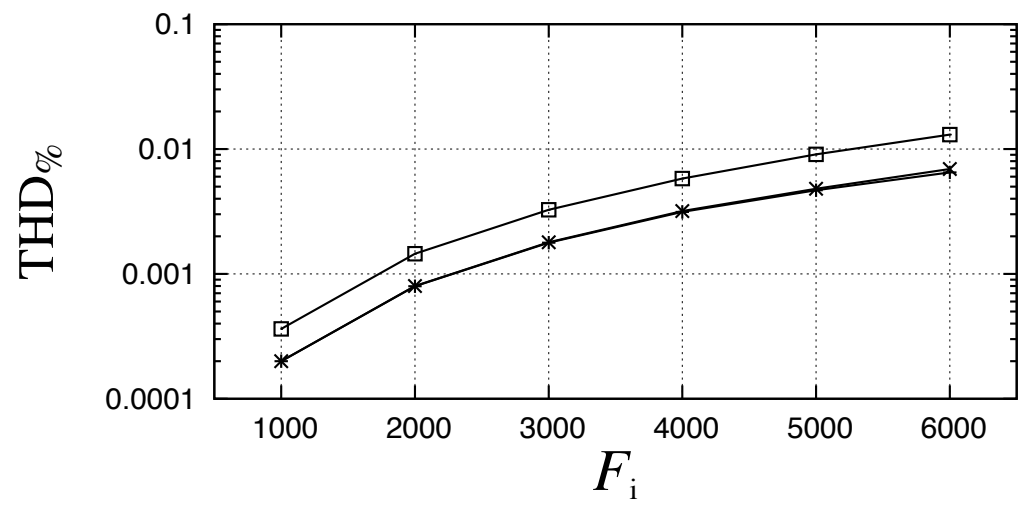

FIG. 4. THD $\%$ against frequency of the input signal, which has modulation index $s_{0}=0.7$. Results from MATLAB simulation and analysis are, respectively, indicated by the symbols + and $\times$ (the difference is apparent only for the highest frequency used here); the symbol $\square$ indicates results for conventional PWM.

the audio range, thus for a $5 \mathrm{kHz}$ input we need include only the third harmonic. MATLAB simulation results for a conventional first-order class-D amplifier are provided for comparison. As illustrated in Fig. 3, the agreement between the analytical results and the MATLAB simulations is excellent. Compared with the conventional PWM topology, the self-oscillating design achieves better linearity at low modulation index, but the advantage reduces with increasing input signal magnitude. This observation is consistent with that reported in Poulsen \& Andersen (2004).

The dependence of the THD on the input signal frequency is illustrated in Fig. 4. Here, the modulation index of the input signal is fixed at $s_{0}=0.7$. The input signal frequency $F_{\mathrm{i}}=\omega /(2 \pi)$ varies from $1 \mathrm{kHz}$ to $6 \mathrm{kHz}$. To a very good approximation, the THD curve varies quadratically with frequency, which represents the same trend as that of a conventional PWM design. This plot also illustrates that the intrinsic distortion of the self-oscillating design is less than that of the conventional PWM design.

\section{Discussion}

It is of interest to compare our results with those of Lu \& Gharpurey (2011), who present a detailed analysis of the amplifier design in Fig. 1, based on the quasi-static assumption of Green \& Williams (1992). The latter authors make the approximation that results derived for DC input may be applied to the case of time-varying audio input. As we have indicated in the Introduction, this is likely to be a reasonable leading-order approximation, in view of the relatively slow time scale of the audio signal (compared with the switching) but cannot be the basis for an entirely systematic analysis.

In fact, the quasi-static approximation proves remarkably good in this case. For example, for the parameters of the previous section, there is less than $0.02 \%$ difference between the leading-order mean switching period in (50) and the first correction in (51), over the range of $s_{0}$.

For the distortion calculation, Lu and Gharpurey calculate the amplitude of the $\sin 3 \omega t$ term in (46) to be (in our notation)

$$
-s_{0} \mathrm{~J}_{1}\left(\omega c T^{2} s_{0}^{2} / 32\right) \omega / \sqrt{c^{2}+\omega^{2}} \sim-\frac{(\omega T)^{2} s_{0}^{3}}{64},
$$


where the right-hand side of this expression results from (i) applying $\omega^{2} \ll c^{2}$ and (ii) Taylor-expanding the Bessel function $\mathrm{J}_{1}$ and keeping the leading term, for consistency with our leading-order contribution to the third-harmonic distortion term in (46). This result relies on their fitting one parameter (their loopgain ratio $G$ ) by simulation. (We note that a quasi-static approach is also used by Risbo \& Neesgaard (2006) to determine the third-harmonic distortion in the fixed-frequency carrier-wave case; unlike the analysis of Lu and Gharpurey, no parameter-fitting is required.) Our corresponding result, from (46), is (with no parameter-fitting necessary)

$$
-\frac{(\omega T)^{2} s_{0}}{8} \frac{\left(1-s_{0}^{2} / 2\right)^{2}\left(1-\left(1-s_{0}^{2}\right)^{1 / 2}\right)}{\left(\left(1-s_{0}^{2}\right)^{1 / 2}+1\right)^{2}} .
$$

The ratio between these quantities turns out to be very close to 1 for reasonable values of $s_{0}$. Indeed, for all $0<s_{0}<0.9$ (where $s_{0}=0.9$ is the maximum value used in $\mathrm{Lu}$ and Gharpurey's simulations) the ratio lies between 0.9 and 1 , corresponding to a difference between our predictions and theirs of less that $1 \mathrm{~dB}$.

Overall, then, the quasi-static approach adopted by $\mathrm{Lu}$ and Gharpurey proves remarkably accurate in this case in characterising the third-harmonic distortion; however, it is not clear that it accurately characterises other components of the distortion. Furthermore, it remains to be seen whether a similar accuracy holds when quasi-static models are applied to other self-oscillating designs. Finally, we emphasise that it is clearly preferable to have a systematic mathematical model (as described in this paper) that is not reliant on parameter-fitting.

\section{Conclusions}

In this paper, we have presented a systematic perturbation analysis of a first-order, hysteresis-controlled, self-oscillating, class-D amplifier. We have determined closed-form expressions for the mean switching period and the principal components of the audio output, both for a general input signal and for the special case of a sinusoidal input. We have corroborated our asymptotic results through demonstrated agreement with simulations.

The general expressions we have derived for (i) the audio output and (ii) the mean switching period provide valuable new tools for engineers. The former is of particular utility because it can be used to calculate the IMD, or other distortion characteristics beyond simply the THD which arises for a sinusoidal input; these broader distortion measures can represent better the extent to which the distortion detracts from the listening experience (Cox et al., 2013; Metzler, 1993; Yu et al., 2012). Similarly, the latter allows more precise determination of the modulation to the switching period for realistic input signals, rather than for a single sinusoid. Our correction to the leading-order result of Lu \& Gharpurey (2011) for the mean switching period appears to make a trivial difference to the result (around 0.02\%), but given the high frequency of oscillation of the output pulse train, even such a small discrepancy can lead to significant intermodulation products in the audio band in stereo applications.

Finally, we note that our treatment possesses significant advantages over prior approaches, in requiring neither a quasi-static approximation nor parameter-fitting. The techniques set out above can clearly be extended to other designs of self-oscillating class-D amplifier or sigma-delta modulator, including higher-order designs. The key steps - the systematic perturbation expansion and the warped-time transformation that renders the demodulation calculation straightforward - provide the means for capturing all the distortion terms at each asymptotic order and for likewise capturing all contributions to the modulation of the switching frequency. 


\section{Fourier coefficients for the audio output}

In Section 3.3, we quoted the Fourier-series coefficients for

$$
Z(x) \equiv \frac{s_{0} \sin x\left(1-2 s_{0}^{2}+s_{0}^{2} \sin ^{2} x\right)}{3\left(1-s_{0}^{2} \sin ^{2} x\right)^{2}} .
$$

Here we derive them. One way to proceed is the following. We note that $Z$ is an odd function and that $Z(\pi-x)=Z(x)$, hence we may write

$$
Z(x)=\sum_{m=0}^{\infty} C_{2 m+1} \sin (2 m+1) x
$$

for some coefficients $C_{2 m+1}$. If we consider the Fourier sine series

$$
P(x)=\sum_{m=0}^{\infty}(2 m+1) p^{m} \sin (2 m+1) x
$$

for some constant $p \in(-1,1)$, we see that

$$
\begin{aligned}
P(x) & =-\mathfrak{R}\left(\frac{\mathrm{d}}{\mathrm{d} x}\left(\mathrm{e}^{\mathrm{i} x} \sum_{m=0}^{\infty} p^{m} \mathrm{e}^{2 \mathrm{i} m x}\right)\right)=-\mathfrak{R}\left(\frac{\mathrm{d}}{\mathrm{d} x} \frac{\mathrm{e}^{\mathrm{i} x}}{1-p \mathrm{e}^{2 \mathrm{i} x}}\right) \\
& =\mathfrak{I}\left(\frac{\left(\mathrm{e}^{-\mathrm{i} x}+p \mathrm{e}^{\mathrm{i} x}\right)\left(\mathrm{e}^{\mathrm{i} x}-p \mathrm{e}^{-\mathrm{i} x}\right)^{2}}{\left|\mathrm{e}^{-\mathrm{i} x}-p \mathrm{e}^{\mathrm{i} x}\right|^{4}}\right)=\frac{(1-p)\left(1+6 p+p^{2}-4 p \sin ^{2} x\right) \sin x}{\left((1-p)^{2}+4 p \sin ^{2} x\right)^{2}} .
\end{aligned}
$$

Then by setting $p=-(1-q) /(1+q)$, where $q=\sqrt{1-s_{0}^{2}}$, we have

$$
P(x)=\frac{(1+q)\left(1-2 s_{0}^{2}+s_{0}^{2} \sin ^{2} x\right) \sin x}{2\left(1-s_{0}^{2} \sin ^{2} x\right)^{2}}=\frac{3(1+q) Z(x)}{2 s_{0}} \text {. }
$$

Hence

$$
C_{2 m+1}=\frac{2(2 m+1)\left(\left(1-s_{0}^{2}\right)^{1 / 2}-1\right)^{m} s_{0}}{3\left(\left(1-s_{0}^{2}\right)^{1 / 2}+1\right)^{m+1}}
$$

as claimed.

\section{References}

Berkhout, M. \& Dooper, L. (2010) Class-D audio amplifiers in mobile applications. IEEE Trans. Circuits Syst. I Regul. Pap., 57, 992-1002.

BLACK, H. S. (1953) Modulation Theory. New York: Van Nostrand.

CHEnG, Y.-H. \& TAN, N.-X. (2011) Third harmonic distortion calculation of a self-oscillating power amplifier. J. Zhejiang University Science C, 12, 307-316.

Cox, S. M. (2009) Voltage and current spectra for a single-phase voltage-source inverter. IMA J. Appl. Math., 74, 782-805. 
Cox, S. M. \& CANDY, B. H. (2005) Class-D audio amplifiers with negative feedback. SIAM J. Appl. Math., 66, 468-488.

Cox, S. M., LAM, C. K. \& TAN, M. T. (2013) A second-order PWM-in/PWM-out class-D audio amplifier. IMA J. Appl. Math., 78, 159-180.

Cox, S. M. \& Mouton, H. Du T. (2015) Ripple compensation for a Class-D amplifier. SIAM J. Appl. Math., 75, 1536-1552.

Cox, S. M., TAN, M. T. \& YU, J. (2011) A second-order class-D amplifier. SIAM J. Appl. Math., 71, 270-287.

Cox, S. M., YU, J., Goh, W. L. \& TAN, M. T. (2013) Intrinsic distortion of a fully differential BD-modulated class-D amplifier with analog feedback. IEEE Trans. Circuits Syst. I Regul. Pap., 60, $63-73$.

Guo, L., GE, T. \& ChAng, J. (2014) Intermodulation distortions of bang-bang control class D amplifiers. IEEE Trans. Power Electronics, 29, 6604-6614.

Green, T. C. \& Williams, B. W. (1992) Spectra of delta-sigma modulated inverters: an analytical treatment. IEEE Trans. Power Electronics, 7, 644-654.

Høyerby, M. C. W. \& Andersen, M. A. E. (2009) Carrier distortion in hysteretic self-oscillating class-D audio power amplifiers: analysis and optimization. IEEE Trans. Power Electronics, 24, 714729.

LU, J. \& GHARPurey, R. (2011) Design and analysis of a self-oscillating class D audio amplifier employing a hysteretic comparator. IEEE J. Solid-State Circuits, 46, 2336-2349.

Metzler, B. (1993) Audio Measurement Handbook. Beaverton: Audio Precision.

Piessens, T. And Steyaert, M. S. J. (2005) Behavioral analysis of self-oscillating class D line drivers. IEEE Trans. Circuits Syst. I Regul. Pap., 52, 706-714.

Pillonnet, G., Cellier, R., Abouchi, N. \& Chiollaz, M. (2008) A high performance switching audio amplifier using sliding mode control. Proceedings of the IEEE North-East Workshop on Circuits and Systems/Analog Information and Signal Processing, and its Applications (NEWCAS-TAISA), IEEE Conference Publications, 305-309.

Poulsen, S. \& Andersen, M. A. E. (2004) Self oscillating PWM modulators, a topological comparision. Proceedings of the IEEE Power Modulator Symposium and 26th High-Voltage Workshop, IEEE Conference Publications, 403-407.

Poulsen, S. \& Andersen, M. A. E. (2005) Hysteresis controller with constant switching frequency. IEEE Trans. Consumer Electronics, 51, 688-693.

Putzeys, B. (2005) Simple self-oscillating class D amplifier with full output filter control. Proceedings of the 118th Audio Engineering Society Convention, Audio Engineering Society, paper number 6453. 
PutZEYs, B. (2009) Globally modulated self-oscillating amplifier with improved linearity. Proceedings of the 37th Audio Engineering Society International Conference, Audio Engineering Society, paper number 4.

RISBo, L. \& NEESGAARD, C. (2006) PWM amplifier control loops with minimum aliasing distortion. Proceedings of the 120th Audio Engineering Society Convention, Audio Engineering Society, paper number 6693.

Tan, M. T., Chang, J. S., Chua, H. C. \& Gwee, B. H. (2003) An investigation into the parameters affecting total harmonic distortion in low-voltage low-power class-D amplifiers. IEEE Trans. Circuits Syst. I Regul. Pap., 50, 1304-1315.

Yu, J., TAN, M. T., Cox, S. M. \& GoH, W. L. (2012) Time-domain analysis of intermodulation distortion of closed-loop class-D amplifiers. IEEE Trans. Power Electronics, 27, 2453-2461.

Yu, W. F., Shu, W. \& Chang, J. S. (2009) A low THD analog class D amplifier based on selfoscillating modulation with complete feedback network. Proceedings of the IEEE International Symposium on Circuits and Systems, IEEE Conference Publications, 2729-2732. 